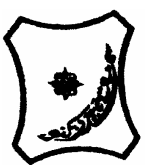

\title{
SYNTHESIS AND CHARACTERISATION OF TEMPERATURE RESPONSIVE POLY (2-ETHYL-2-OXAZOLINES)
}

\author{
Haruna, $M$. \\ Department of Pure \& Industrial Chemistry, Bayero University, P.M.B. 3011, Kano, Nigeria \\ hdambatta@yahoo.co.uk
}

\begin{abstract}
A bifunctional Poly(2-ethyl-2-oxazolines) containing hydrophobic end groups at both end of the polymer chains were synthesised by terminating a living cationic polymerisation with 4bromomethyl benzoic acid. The results obtained for the clouding point measurement using UVvisible spectrophotometer shows that bifunctional polymer containing 20 monomer units (DP 20) exhibited a lower critical solution temperature (LCST) near $49^{\circ} \mathrm{C}$ due to formation of hydrophobic domains by the polymer end-groups. In contrast, the polymer with 50 monomer units does not exhibit any LCST between 20 to $70^{\circ} \mathrm{C}$ due to the imbalance in the hydrophilic-hydrophobic interaction in this polymer.
\end{abstract}
Keywords: Cationic
polymerisation,
end-groups,
Poly(2-ethyl-2-oxazolines),
LCST, Thermoresponsive

\section{INTRODUCTION}

Polymers from 2-oxazolines have found various uses as resins, gelling agents, thickening agents, adhesives of tyre cords, antistatic agents, surface coatings and as hard component for thermosetting rubber. They are also useful starting materials for the preparation

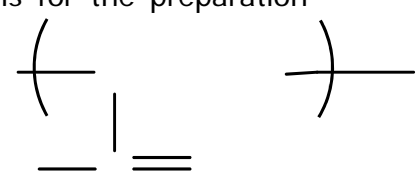

of linear polyalkylenimines which exhibits the ability to form complexes. This makes them useful for heavy metal adsorption and removal (Kobayashi and Saegusa, 1984).

\section{Figure 1: General structure of Poly (2-alkyl-2-oxazolines) ( $\mathbf{R}=$ alkyl group)}

Thermoresponsive polymers are polymers that undergo large change in properties in response to changes in temperature and are currently receiving greater attention in view of their variety of applications in medicine and biotechnology. Among the most widely studied Thermoresponsive polymers is Poly(N-isopropyl acrylamide) (PNIPAM) which has a lower critical solution temperature (LCST) of $32^{\circ} \mathrm{C}$. The LCST is the transition temperature at which a polymer is insoluble in a good solvent and undergoes phase separation from solution while below LCST, the polymer is miscible in all proportion. However, the major disadvantage of PNIPAM is it undergoes strong hysteresis due to the formation of intramolecular hydrogen bonds in the collapsed state, hence Poly(2oxazolines) could be a better alternative to PNIPAM because they exhibit a reversible, concentration dependent phase separation and do not show hysteresis (Hoogenboom et al., 2008).

Several authors have synthesised poly(2oxazolines) and their copolymers by living cationic polymerisation as well investigating their Thermoresponsive behaviour in aqueous media. The synthesis and characterisation of Linear poly(2-alkyl-2oxazolines) having an acrylate end groups, narrow molecular weight distribution and well controlled molecular weight were synthesised by terminating a living, bifunctional polymer with acrylic acid (Christova et al, 1997). Similarly, temperature sensitive hydrogels have been prepared based on combination of poly(2ethyl-2-oxazoline) (PEtOx) with other polymers e.g. poly(HEMA), PMMA etc and it was observed that the LCST behaviour of the water swollen hydrogels can be tuned by varying the poly(2-ethyl-2-oxazoline) content and the amount of hydrophobic comonomer used (Christova et al., 2003)

The synthesis of several end-functionalized poly(2-isopropyl-2-oxazolines) has been carried out by cationic ring-opening polymerization of 2-isopropyl-2 oxazoline. These polymers possess well-controlled molecular weight, low polydispersity and exhibited an LCST near $37^{\circ} \mathrm{C}$ which suggests promising applications as bioconjugates, hydrogels, and drug carriers (Park, et al, 2004). Most of the poly(2-alkyl-2-oxazoline)s have been shown to exhibit temperature induced phase separation and the cloud point temperature depends on the molecular weight and concentration of the polymers (Chiu, et al., 1986)

The effect of temperature on aqueous solutions of poly (2-isopropyl-2-oxazoline) samples of molecular weights ranging from 1900 to $5700 \mathrm{~g} \mathrm{~mol}^{-1}$ was studied by turbidimetry and calorimetry in a temperature range of 10 to $80^{\circ} \mathrm{C}$. 
Depending on the molecular weight of the polymers, they were soluble in cold water and showed phase separation at cloud point temperature $\left(T_{c p}\right)$ between $45-63^{\circ} \mathrm{C}$ (Diab et al, 2004). Well-defined gradient or random copolymers of 2-n-propyl-2-oxazoline and either 2-isopropyl-2-oxazoline or 2-ethyl-2-oxazoline have been synthesised. The LCST of these polymers was found to change by varying the ratio between hydrophilic and hydrophobic monomers (Park and Kataoka, 2007). Furthermore, it has been demonstrated that the LCST of amphiphilic poly(2isopropyl-2-oxazoline) gradient copolymers can be precisely tuned via the copolymerization with 2-ethyl2-oxazoline as the hydrophilic comonomer portion. These copolymers show a linear increase in LCST with an increasing amount of 2-ethyl-oxazoline comonomer (Park and Kataoka, 2006).

In this investigation, 1,4-dibromo-2-butene and allyl bromide were used as cationic initiators for the synthesis of bifunctional and monofunctional poly(2-ethyl-2-oxazolines) respectively. Moreover, alpha-bromo-p-toluic acid was used to terminate these polymerisations in order to introduce some endgroups in the polymer and their LCST were investigated.

\section{MATERIALS AND METHODS}

2-Ethyl-2-oxazoline (EtOx) (Aldrich, 99+ \%) was refluxed over $\mathrm{CaH}_{2} / \mathrm{KOH}$ and distilled. 1, 4-Dibromo-2butene (DBB) (Aldrich, 99\%) was dried in vacuum before use. Acetonitrile (Aldrich, 99.9+ \%) was purified by distillation over calcium hydride, Triethylamine $\left(\mathrm{Et}_{3} \mathrm{~N}\right)$ and 2, 6-lutidine were refluxed over $\mathrm{CaH}_{2}$ and distilled, 4-bromomethyl benzoic acid (Aldrich, 99+ \%). All purification operations were carried out under nitrogen. Other reagents and solvents were of high purity and used as received.

\section{SYNTHESIS}

\section{Synthesis of bifunctional Poly(2-ethyl-2- oxazoline) (Theoretical DP 20) by Controlled/living Cationic Polymerisation}

A mixture of $0.543 \mathrm{gm}(2.57 \mathrm{mmol})$ of 1,4-Dibromo-2butene and $5.092 \mathrm{~g}(51.36 \mathrm{mmol})$ of 2-ethyl-2oxazoline in $20 \mathrm{ml}$ of $\mathrm{CH}_{3} \mathrm{CN}$ was stirred at $70^{\circ} \mathrm{C}$ for 7 hours. After cooling to $0^{\circ} \mathrm{C}, 2.76 \mathrm{gm}(12.83 \mathrm{mmol})$ of 4-bromomethyl benzoic acid (terminating agent) and $1.376 \mathrm{~g}(12.84 \mathrm{mmol})$ of 2,6-lutidine were added and the reaction mixture was stirred at $60^{\circ} \mathrm{C}$ for $24 \mathrm{hrs}$. The mixture was cooled to room temperature, poured into $300 \mathrm{ml}$ of cooled diethyl ether and the polymeric product was collected and dried under vacuum overnight. To remove the unreacted terminating agent, the product was dissolved in $100 \mathrm{~mL}$ methanol and stirred with $4 \mathrm{~g}$ of Amberlyst-15 ion exchange resin at room temperature for $24 \mathrm{~h}$. After filtrating off the ion-exchange resin, the filtrate was concentrated in vacuum and precipitated in cooled diethyl ether. The macroinitiator thus obtained was purified by reprecipitation from methanol solution in diethyl ether and dried in vacuum. The yield of the product obtained was $70 \%$.

The ${ }^{1} \mathrm{H}$ NMR of the polymer in Deuterated chloroform $\left(\mathrm{CDCl}_{3}\right)$ was acquired using Varian $400 \mathrm{MHz} \mathrm{NMR}$ spectrophotometer. The spectrum is presented in Figure III

\section{Synthesis of bifunctional Poly(2-ethyl-2- oxazoline) (Theoretical DP 50) by Controlled/living cationic polymerisation}

A mixture of $0.221 \mathrm{gm}(1.029 \mathrm{mmol})$ of 1,4-Dibromo2- butene and $5.092 \mathrm{~g}$ ( $51.36 \mathrm{mmol})$ of 2-ethyl-2oxazoline in $20 \mathrm{~mL}$ of $\mathrm{CH}_{3} \mathrm{CN}$ was stirred at $70^{\circ} \mathrm{C}$ for 7 hours. After cooling to $0^{\circ} \mathrm{C}, 1.108 \mathrm{gm}(5.15 \mathrm{mmol})$ of 4-bromomethyl benzoic acid (terminating agent) and $0.552 \mathrm{~g}(5.15 \mathrm{mmol})$ of 2,6-lutidine were added and the reaction mixture was stirred at $60^{\circ} \mathrm{C}$ for $24 \mathrm{~h}$. The mixture was cooled to room temperature, poured into $300 \mathrm{ml}$ of cooled diethyl ether and the polymeric product was collected and dried under vacuum overnight. To remove unreacted terminating agent, the product was dissolved in $100 \mathrm{~mL}$ methanol and stirred with $4 \mathrm{~g}$ of Amberlyst-15 ion exchange resin at room temperature for $24 \mathrm{~h}$. After filtrating off the ionexchange resin, the filtrate was concentrated in vacuum and precipitated in cooled diethyl ether. The macroinitiator thus obtained was purified by reprecipitation from methanol solution in diethyl ether and dried in vacuum. The yield of the product obtained was $70 \%$. The ${ }^{1} \mathrm{H}$ NMR of the polymer in Deuterated chloroform $\left(\mathrm{CDCl}_{3}\right)$ was acquired using Varian $400 \mathrm{MHz}$ NMR spectrophotometer. The spectrum is presented in Figure IV.

\section{Determination of lower critical solution temperature (LCST) of poly(2-ethyl-2- oxazoline)}

$4 \mathrm{mg} / \mathrm{ml}$ of polymer solutions were made in distilled water. The solutions were stored in the fridge for 1 week, and then filtered in order to remove impurities such as lutidinium salt that could interfere with the cloud point temperature measurements. The transmittances of the solutions were acquired as a function of temperature during heating/cooling cycles on a HP/Agilent 8453 UV-visible spectrophotometer equipped with temperature control at $500 \mathrm{~nm}$. A temperature interval of $1^{\circ} \mathrm{C}$ was used for all the measurements and the samples were allowed to equilibrate for 5 minutes prior to each measurement. The LCST was defined as the temperature corresponding to a $50 \%$ decrease in the initial value of transmittance at $500 \mathrm{~nm}$ and was estimated from the averages of cloud point temperatures obtained for the heating and cooling curves.

\section{RESULTS AND DISCUSSION}

Poly(2-alkyl-2-oxazolines) are generally synthesised by cationic ring opening polymerisation of 2-alkyl-2oxazoline monomers. The mechanism involved in this reaction is illustrated in Figure 2. 


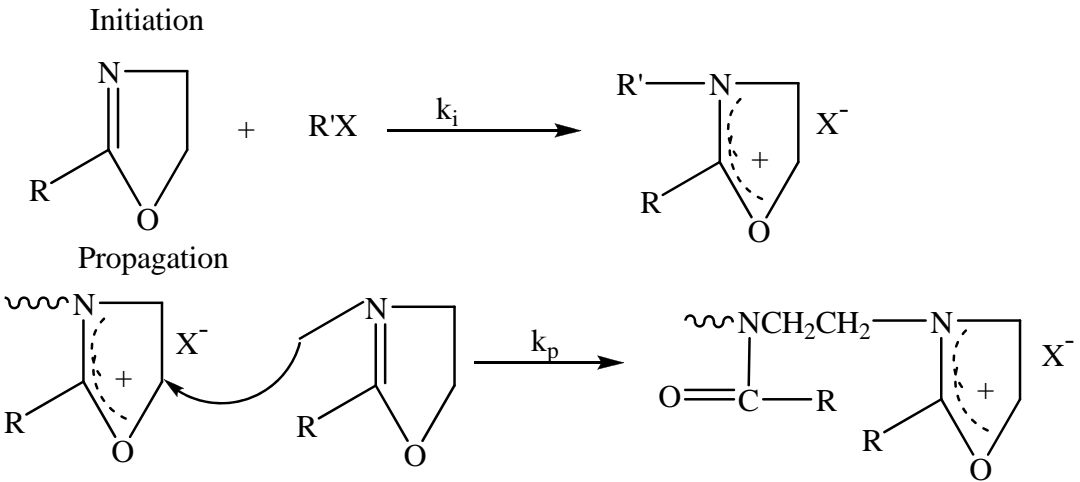

Figure 2: Reaction mechanism for the cationic polymerisation of 2-alkyl-2-oxazo- lines ( $R$ 'X is an initiator having a weakly nucleophilic $\left.\mathbf{X}^{-}\right)^{1}$.

One advantage of controlled/living cationic polymerisation is that compounds containing hydrophobic end groups can be used to terminate a reaction. The type of polymer produced (e.g. monofunctional or bifunctional) depends on the nature of cationic initiator used at the initiation stage of the controlled living cationic polymerisation. In this investigation, 1,4-dibromobutene was employed as initiator in order to produce a bifunctional poly(2ethyl-2-oxazoline) which was subsequently terminated with 4-bromomethyl benzoic acid to produce a polymer with hydrophobic end-group at both end of the chain (bifunctional). ${ }^{1} \mathrm{H}$ NMR of the polymer has confirmed the formation of this compound and the spectrum is presented in Figures 3 and 4.

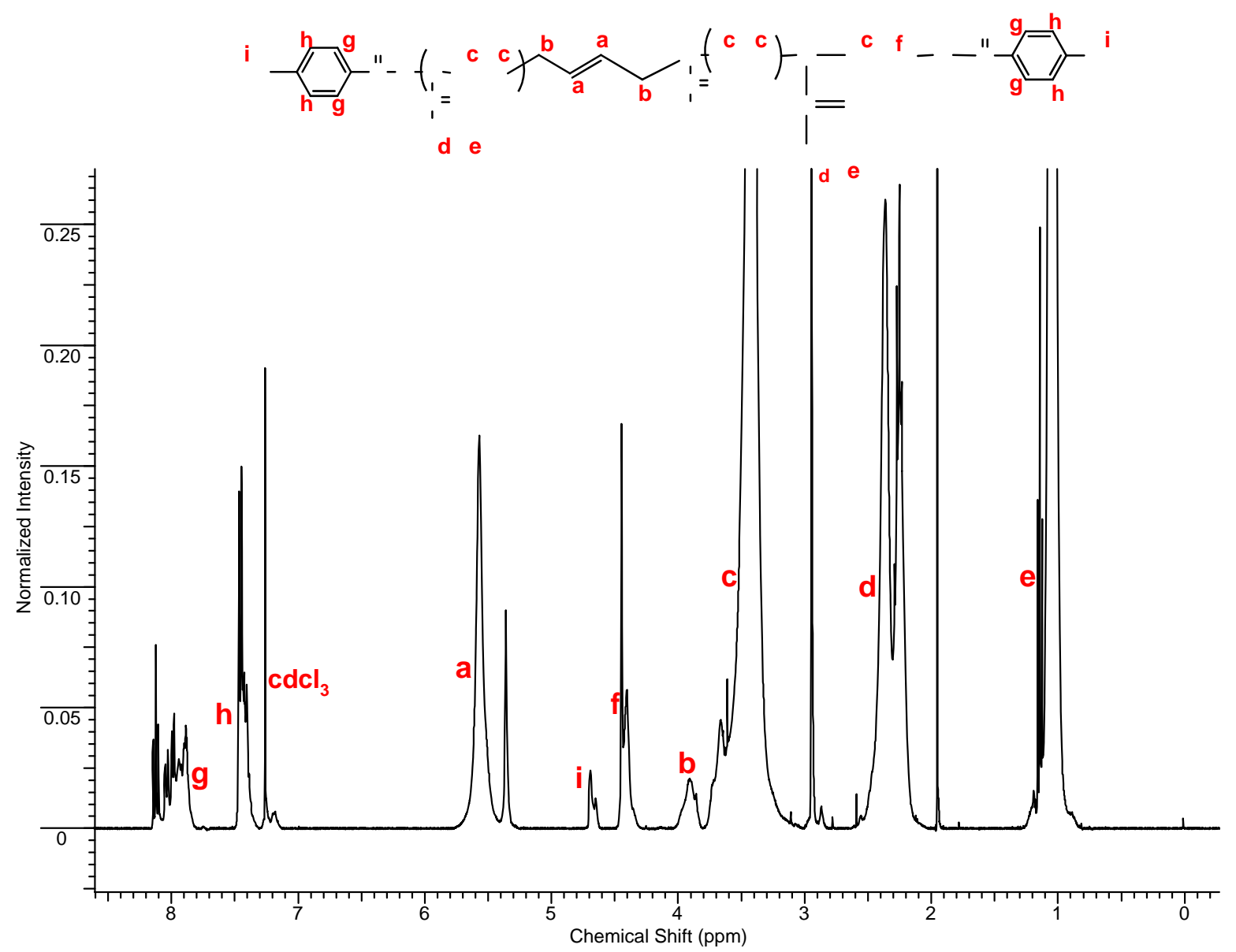

Figure 3: ${ }^{1} \mathrm{H}$ NMR of bifunctional poly(2-ethyl-2-oxazoline) (PEtOx-1) in $\mathrm{CDCl}_{3}$ : $\delta$ ppm; 7.98 (d, $\mathrm{H}$

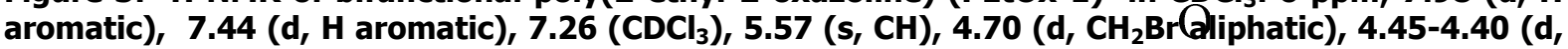

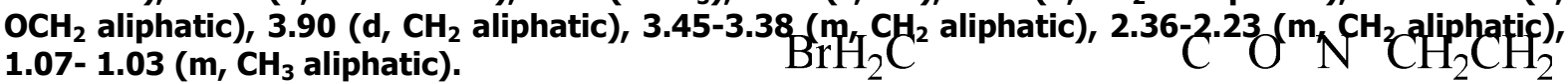

$\mathrm{C} \mathrm{O}$ 


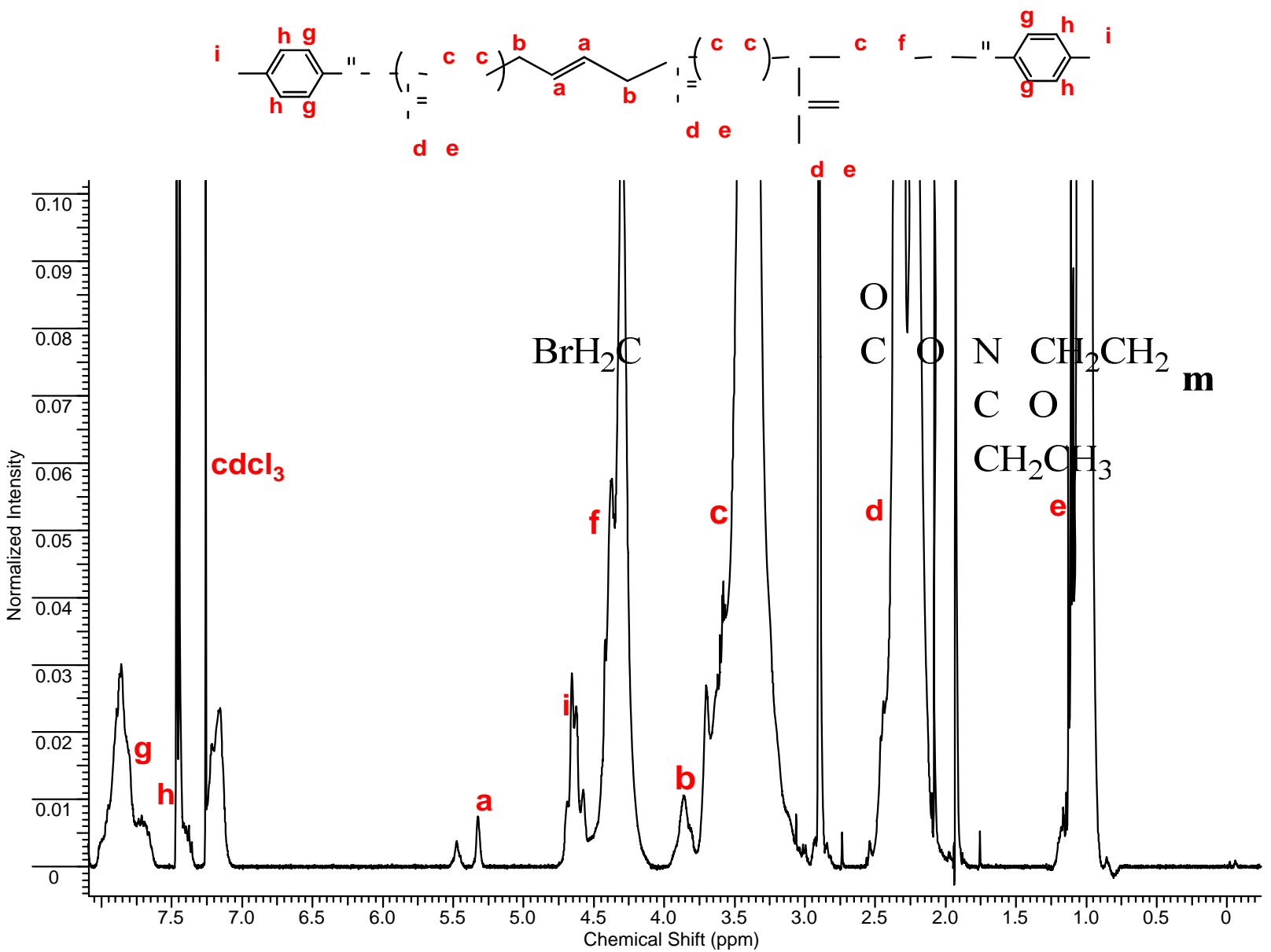

Figure 4: ${ }^{1} \mathrm{H}$ NMR of bifunctional poly(2-ethyl-2-oxazoline) (PEtOx-2) in $\mathrm{CDCl}_{3}$ : $\mathbf{~ p p m ; ~} 7.89$ (d, $\mathrm{H}$ aromatic), 7.44 (d, H aromatic), $7.26\left(\mathrm{CDCl}_{3}\right), 5.30(\mathrm{~s}, \mathrm{CH}), 4.66$ (d, $\mathrm{CH}_{2} \mathrm{Br}$ aliphatic), 4.42-4.30 (d, $\mathrm{OCH}_{2}$ aliphatic), 3.86 (d, $\mathrm{CH}_{2}$ aliphatic), 3.40-3.34 ( $\mathrm{m}, \mathrm{CH}_{2}$ aliphatic), 2.33-2.22 (m, $\mathrm{CH}_{2}$ aliphatic), 1.13-1.02 ( $\mathrm{m}, \mathrm{CH}_{3}$ aliphatic).

The sharp peak observed at a chemical shift around 2 ppm was due to residual Acetonitrile that was used as polymerisation solvent and the other peak that occurs at $3 \mathrm{ppm}$ was due to traces of diethyl ether that was used as non-solvent to precipitate the polymer at the end of the polymerisation. Hence the polymers were further dried under vacuum prior to LCST measurements.

\section{Lower Critical Solution Temperature (LCST) of Poly(2-alkyl-2-oxazoline)}

Poly(2-alkyl-2-oxazolines) are very important class of Thermoresponsive polymers that exhibit an LCST above which the polymer is insoluble in water and precipitates and below which the polymer is soluble and gives a clear homogenous solution. This property and a host of others make this polymer potentially useful in biomedical applications Generally, the LCST of poly(2-alkyl-2oxazolines) are very much dependent on the chain length, hence the molecular weight of polymers as illustrated by (Christova et al , 2003) and (Park, et al, 2004).

The digital images obtained after the heating and cooling cycles of the Poly(2-ethyl-2-oxazolines) samples in water are presented in Figure V. PEtOx-1 (Sample1) bifunctional polymer (DP 20) exhibited a reversible Thermoresponsive property where their solution reversibly changes from clear to a very cloudy appearance in response to changes in temperature. On the other hand, PEtOx-2 bifunctional macroinitiator (DP 50) does not respond to any changes in the solution temperature as shown by Sample 2 in Figure 5.

Similarly, the results obtained for the clouding point measurement of the poly(2-ethyl-2-oxazolines) shows that PEtOx-1 bifunctional polymer (DP 20) exhibited an LCST near $49^{\circ} \mathrm{C}$ which is in contrast with earlier reports that suggested a minimum DP of 100 is required for this polymer to show an LCST (Hoogenboom, et al, 2008). Therefore, the LCST exhibited by this polymer can be ascribed to the presence of 2 hydrophobic benzyl bromide end-groups present on both ends of polymer chain. These hydrophobic end groups could associate, forming hydrophobic domains which resulted in a reversible LCST behaviour as shown in the cooling and heating curves in Figure 4. However, PEtOx-2 bifunctional polymer (DP 50) does not exhibit any LCST between 20 to $70^{\circ} \mathrm{C}$ due to the imbalance in the hydrophilic-hydrophobic interaction in this polymer. The presence of 50 hydrophilic 2-oxazolines units make this polymer highly hydrophilic and therefore the hydrophobic effect exerted by the 2 benzyl bromide end-groups is negligible. The Clouding point curve was obtained by plotting the \% Transmission against Temperature $\left({ }^{\circ} \mathrm{C}\right)$. From this curve, the LCST was estimated. 


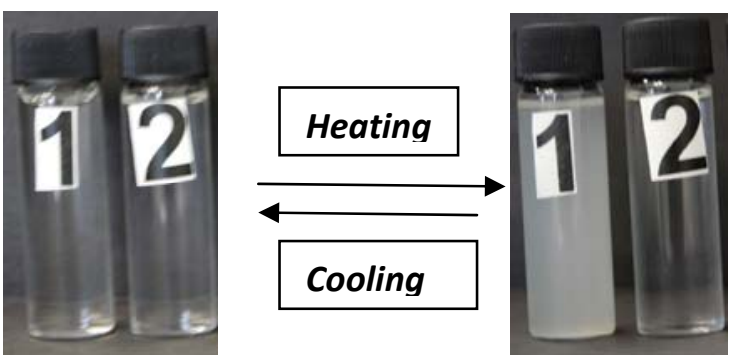

Figure 5: Digital images of PEtOx-1 and PEtOx-2 after the heating and cooling cycles

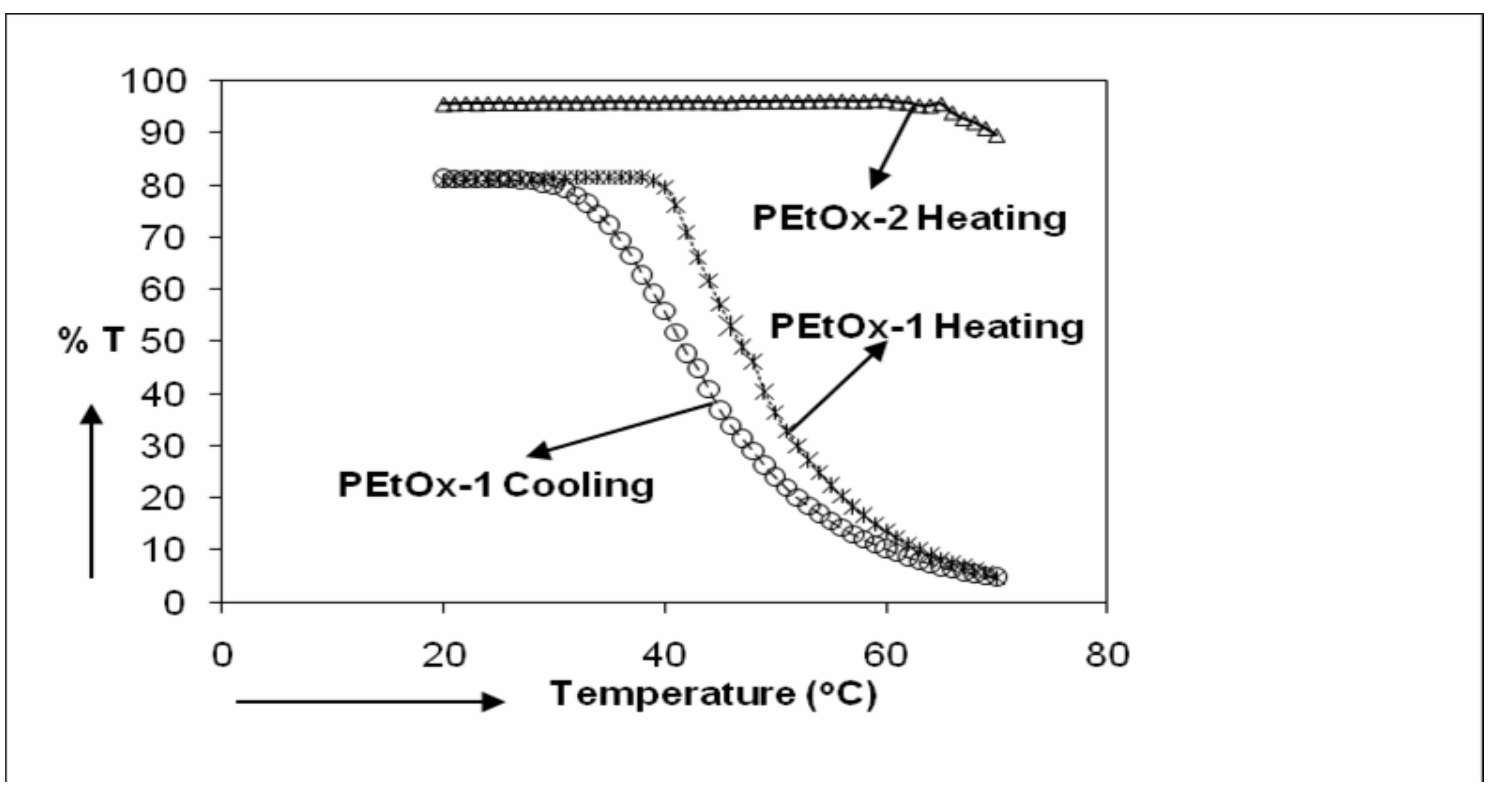

Figure 4: Clouding Point curve of bifunctional PEtOx-1 and PEtOx-2 after Heating and Cooling at Temperatures between $20-70^{\circ} \mathrm{C}$

\section{Conclusion}

Temperature responsive end-functional poly(2-ethyl-2oxazoline) have been synthesised by living cationic polymerisation as confirmed by ${ }^{1} \mathrm{H}$ NMR. The lower critical solution temperatures (LCST) exhibited by these polymers depend on the balance between the hydrophilic

\section{REFERENCES}

Chiu, T. T; Thill, B. P; Fairchok W. J. (1986), In: Glass, J. E., editor. Water Soluble Polymers. Advances in Chemistry Series 213, Washington: American Chemical Society. pg. 425-433.

Christova, D.; Velichkova, R.; Goethals, E. J. (1997), Bismacromonomers of 2-alkyl-2-oxazolines synthesis and polymerization, Macromol. Rapid. Commun. 18, 1067-1073.

Christova, D.; Velichkova, R.; Loos, W.; Goethals, E. J.; Du Prez, F., (2003), New thermo-responsive polymer materials based on poly(2-ethyl-2oxazoline) segments, Polymer, 44, 2255-2261.

Diab, C.; Akiyama, Y.; Kataoka K.; Winnik, F. M. (2004), Microcalorimetric Study of the TemperatureInduced Phase Separation in Aqueous Solutions of Poly (2-isopropyl-2-oxazolines), Macromolecules 37, 2556-2562.

Hoogenboom, R.; Thijs, H. M. L.; Jochems, M. J. H. C.; van Lankvelt, B. M.; Fijtena, M. W. M.; Schubert, U. S. (2008), Tuning the LCST of poly(2-oxazoline)s by varying composition and polymer segments and the hydrophobic end-groups present in the polymers.

\section{Acknowledgement}

The Author thank Bayero University Kano for Study Fellowship, Mac-Arthur Foundation and Kano State Government for funding my PhD studies.

molecular weight: alternatives to $\operatorname{poly}(\mathrm{N}-$ isopropylacrylamide), Chem. Commun, 57585760.

Kobayashi, S.; Saegusa, T. (1984). Ring opening polymerisation, vol. 2, Elsevier, Applied Science Publishers Ltd.

Park, J-S.; Akiyama, Y.; Winnik, F. M.; Kataoka, K. (2004), Versatile Synthesis of EndFunctionalized Thermosensitive Poly(2isopropyl-2-oxazolines, Macromolecules, 37, 6786-6792.

Park, J-S.; Kataoka, K. (2006), Precise Control of Lower Critical Solution Temperature of Thermosensitive Poly(2-isopropyl-2-oxazoline) via Gradient Copolymerization with 2-Ethyl-2oxazoline as a Hydrophilic Comonomer, Macromolecules, 39, 6622-6630.

Park, J-S.; Kataoka, K. (2007), Comprehensive and Accurate Control of Thermosensitivity of Poly(2alkyl-2-oxazoline)s via Well-Defined Gradient or Random Copolymerization Macromolecules 40, 3599-3609. 